\title{
ChemComm
}

Check for updates

Cite this: Chem. Commun., 2019,

55, 11029

Received 19th July 2019

Accepted 20th August 2019

DOI: $10.1039 / c 9 c c 05329 h$

rsc.li/chemcomm

\section{A self-sustained soft actuator able to rock and rollt}

\author{
Marina Pilz da Cunha, (D) ab Akhil R. Peeketi, (D) ${ }^{c}$ Kanishk Mehta, ${ }^{c}$ Dirk J. Broer, (D) ab \\ Ratna K. Annabattula, (D) *c Albert P. H. J. Schenning (iD *ab and \\ Michael G. Debije (ID *ab
}

\begin{abstract}
A triangular shaped liquid crystal network is shown to undergo a continual, rocking chair-like oscillatory chaotic motion upon exposure to a surface of constant temperature. With the addition of an azobenzene chromophore, dual response is achieved, extending the actuation freedom towards a film that shows rocking and rolling motion.
\end{abstract}

Materials that undergo stimuli-triggered and self-sustained oscillatory motion are extensively pursued for applications in medicine and soft robotics. ${ }^{1,2}$ Self-oscillating systems in nature, including the heartbeat, are a constant inspiration for the development of advanced stimuli-responsive materials. ${ }^{3}$ Autonomous motions in polymers have been realized by utilizing variations in ambient humidity, ${ }^{4,5}$ light, ${ }^{6}$ chemo-mechano-chemical effects ${ }^{7}$ and oscillating chemical reactions in gels such as the Belousov Zhabotinsky (BZ) reaction. ${ }^{8,9}$ The continuous self-sustained motion in polymers frees the devices from the dependence of on/off switching of external stimuli, and could dramatically expand the range of applications for soft actuators, including in the areas of autonomous motors ${ }^{10}$ and artificial muscles. ${ }^{11}$ Selfsustained motions in polymer actuators are often a result of feedback loops that couple material properties and external stimuli in synchronism.

The versatility of LCNs has allowed for the development of soft actuators capable of bending, ${ }^{12-14}$ rolling, ${ }^{15}$ 'walking, ${ }^{16-18}$ and helical twisting in a dry environment. ${ }^{19,20}$ Over the past few decades light, ${ }^{21-23}$ heat $^{12}$ and humidity ${ }^{24}$ have been used as the

\footnotetext{
${ }^{a}$ Laboratory of Stimuli-responsive Functional Materials \& Devices, Department of Chemical Engineering and Chemistry,

Eindhoven University of Technology, P.O. Box 513, 5600 MB, Eindhoven, The Netherlands. E-mail: a.p.h.j.schenning@tue.nl, m.g.debije@tue.nl

${ }^{b}$ Institute for Complex Molecular Systems (ICMS),

Eindhoven University of Technology (TU/e), 5600 MB Eindhoven, The Netherlands ${ }^{c}$ Stimuli-Responsive Systems Laboratory, Department of Mechanical Engineering, Indian Institute of Technology Madras (IITM), 600036 Chennai, India.

E-mail: ratna@iitm.ac.in

$\dagger$ Electronic supplementary information (ESI) available. See DOI: 10.1039/ c9cc05329h
}

main triggers. In LCNs, feedback loops arising from humidity variations ${ }^{25}$ or light-triggered deformation and self-shadowing have been exploited to generate oscillatory motion upon exposure to a constant high intensity light ${ }^{6,26-29}$ and have led to the design of continuous walkers/rollers. ${ }^{16}$ Even though the reported light triggered oscillations in LCN cantilevers are rooted in photothermal deformations and self-shadowing, solely thermally-fueled continuous motion is still undocumented for LCN actuators. Fully temperature-triggered continuous actuation was documented for a semi-crystalline random copolymer upon exposure to a heated surface. ${ }^{30}$ Upon partial phase transition, a thermo-mechano feedback loop is generated which results in the self-sustained motion of the material. More recently, a thermo-mechano electrical bilayer system was reported consisting of a three-dimensionally aligned ferroelectric polyvinylidene fluoride (PVDF) and polydopamine modified reduced graphene oxide-carbon nanotube layer capable of autonomous motion driven by heat. ${ }^{31}$

Here, we present a free-standing LC actuator demonstrating unexpected self-sustained oscillating motion initiated by contact with a heated surface as the only stimulus, Fig. 1A and C. We document geometry-specific motions for the polymer film, with triangular and parallelepiped shapes exhibiting unusual selfsustained oscillations resembling a rocking chair, as the polymer film oscillates between two extreme states, rocking from tip to base in an irregular fashion. By the example of the triangular shaped film and through a finite element analysis, we propose the mechanism for the self-oscillation of the material with an insight into factors affecting the movement. Furthermore, with the addition of an azobenzene chromophore in the LCN, dual light/heat response is achieved, extending the actuation freedom of the material towards polymer films that show rocking and rolling motion.

The anisotropic shape of LC molecules can be utilized to create macroscopic deformations in polymer films through controlled molecular alignments. ${ }^{12}$ For the actuator presented here, a self-assembling, splay aligned triangle shaped LCN is used with one side aligned parallel to the surface and the other perpendicular (planar and homeotropic alignment, respectively), see Fig. 1A. 
A

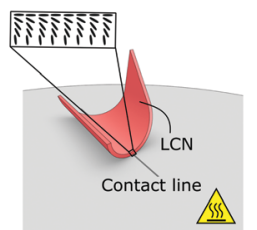

C

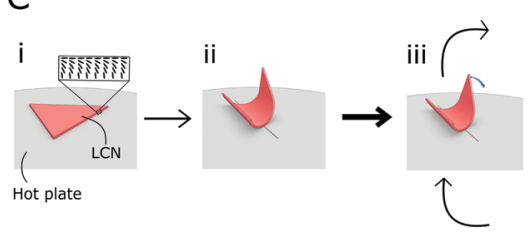

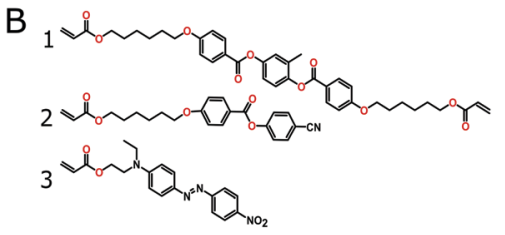

iv

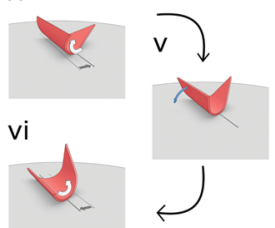

Fig. 1 (A) Schematic of the splay aligned LC film with equilateral triangular shape (sides of $30 \mathrm{~mm}$ ) on a heated surface at $70{ }^{\circ} \mathrm{C}$. (B) Chemical structures of reactive mesogens $(1,2)$ and the azobenzene derivative $(3)$ in the LCN with splay molecular alignment. (C) Schematic depiction of feedback loop for the self-sustained rocking motion on a heated surface. Deformations i and ii are initial deformations before the feedback loop commences.

The LCN is produced by photopolymerization of two LC monomers, a monoacrylate, 2, (40.5 mol\%) and a diacrylate, 1, (56.5 mol\%). Light responsivity was achieved with the addition of a commercially available photothermal azobenzene chromophore with a fast cis-trans isomerization, 3 (see Fig. 1B). The LCN is produced by filling custom-made cells composed of two glass slides coated with differing polyimide layers (one slide planar, the other homeotropic), see ESI $\dagger$ for experimental details. The glass slides are separated by $20 \mu \mathrm{m}$ diameter glass bead spacers incorporated into the glue to achieve controlled cell thickness. Cells filling is done at $95{ }^{\circ} \mathrm{C}$, at which the LC mixture is isotropic and after cooling the polymer film is polymerized at $80{ }^{\circ} \mathrm{C}$, at which temperature the LC mixture is nematic (see Fig. S1, ESI $\dagger$ ). The elevated polymerization temperature gives the films a pre-bent shape at room temperature due to increased molecular order at lower temperature: upon cooling to room temperature from the more disordered state, the molecular order increase leads to contraction perpendicular to the molecular director and expansion parallel to the molecular director (Fig. S2, ESI $\dagger$ ); this causes the homeotropic side of the splay to be on the inside of the film's pre-bent shape. Subsequent thermal treatment at $120{ }^{\circ} \mathrm{C}$ for 10 minutes releases thermal stresses arising from polymer shrinkage during polymerization. After polymerization, the cell was opened, and the films are peeled from the glass.

Curiously, we observe a thermally fueled oscillatory motion when the equilateral triangle splay film is placed with its homeotropic surface in contact with a hot plate set between 70-80 ${ }^{\circ} \mathrm{C}$. Upon contact with the heated surface, the film's extremities are lifted off the heated surface, creating a curved geometry in which a localized contact line is established between the film and the hot plate, Fig. 1C (i-ii). Subsequently, a rapid back-and-forth rocking motion commences and is sustained, Movie S1 (ESI $\dagger$ ) and Fig. 1C (iii-vi). Further increasing the temperature to the material's glass transition temperature
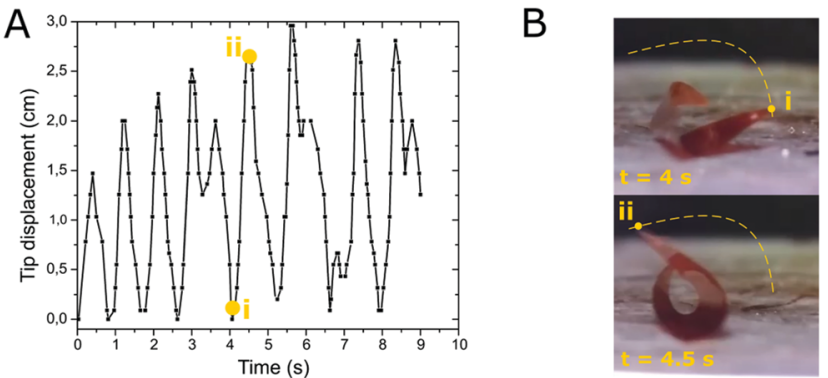

Fig. 2 (A) Plot tracking triangle's tip displacement after the film has initiated oscillatory motion. The tip's path follows the dashed yellow line in the snapshot in (B), with points $\mathrm{i}$ and ii showing the two displacement extremes during an oscillation cycle. (B) Snapshots of one actuation cycle in which the tip moves away from the heated surface following a curved path, (i-ii); the hot plate temperature is set to $72{ }^{\circ} \mathrm{C}$.

$\left(83{ }^{\circ} \mathrm{C}\right)$ stops the continual motion as the material undergoes plastic deformation. Also, no rocking motion is observed when the heated surface is below $70{ }^{\circ} \mathrm{C}$; at a temperature between 45-70 ${ }^{\circ} \mathrm{C}$ the extremities of the film raise above the surface, but no oscillatory motion is initiated and when the temperature is below $45{ }^{\circ} \mathrm{C}$ no consequential motion is observed. When placed with its planar side contacting the hot plate (between $70-80{ }^{\circ} \mathrm{C}$ ), the film uncurls and straightens onto the surface, showing no further motion, Fig. S3 (ESI $\dagger$ ). More information on the nature of thermally induced oscillations is obtained by tracking the displacement of one of the film's extremities in time. Tracking of the triangular film's tip displacement when the film initiates oscillatory motion, (Fig. 2) shows that the rocking motion does not follow an obvious harmonic motion; instead displaying a semi-random displacement. During rocking motion, the film oscillates from two extreme positions and the triangular shape shows different degrees of curvature at each extreme. Irregularities in the displacement are more commonly observed during the reverse motion. The forward motion, from point $\mathrm{i}$ to ii in Fig. 2B, is more consistent with an average tip speed of $7.2 \mathrm{~cm} \mathrm{~s}^{-1}( \pm 17 \%)$.

We have observed that the geometry of the LCN film is important for the oscillatory motion to be sustained. A rectangular or square splay film of the same composition deforms similarly to the triangular film upon initial contact of the homeotropic surface with the hot plate between $70-80{ }^{\circ} \mathrm{C}$, with the edges lifted off the surface and the establishment of a contact line. However, the subsequent deformation results in a toppling of the film onto the planar surface which will not initiate further motions and the film remains nearly flat on the surface, Movie S2 (ESI $\dagger$ ). Despite the similar geometry to a rectangle, a parallelepiped (with similar dimensions), does not show immediate toppling but oscillatory motion similar to the triangular shape, Movie S3 (ESI $\dagger$ ). The oscillatory motion of shape-specific LCNs is rooted in both the temperature-triggered shape deformations and in a steep thermal gradient between the contact line of the film with the hot plate and it's raised extremities. We propose that after establishment of the contact line between the film and the heated surface, the geometry of the raised extremities cannot be mirror images of each other for oscillation, as is the case for a rectangular/square film. For a parallelepiped, comparison across the contact line 
does not present a mirror image of the raised extremities and most likely this condition prevents immediate toppling of the film and results in rocking motion.

Thermal actuation of the polymer film in an oven from room temperature to $90{ }^{\circ} \mathrm{C}$ leads to a reversible thermally driven deformation with three main deformation shapes: below, at, and above $T_{\mathrm{f}}$, Fig. S4A (ESI $\dagger$ ). At room temperature, the network is bent, with the homeotropic side of the splay on the inside of the curl; upon heating the network, the film extends to a flat shape (observed at $60-65{ }^{\circ} \mathrm{C}$ ), followed by bending in the opposite direction, with the planar side now inside the curl (Fig. S4B, ESI $\dagger$ ). Upon heating the LCN, one side displays volumetric expansion parallel to the surface (homeotropic side), and the other anisotropic contraction (planar side), resulting in macroscopic bending. Thermal imaging reveals that while the triangular film undergoes oscillatory motion on a hot plate, tipping from tip to base, steep thermal gradients are present along the film's length, Fig. S4C (ESI $\dagger$ ). The temperature variations follow a linear decrease with increasing distance from the contact line, going from above $70{ }^{\circ} \mathrm{C}$ at the contact line to $33{ }^{\circ} \mathrm{C}$ at the tip's extremity, Fig. S4D (ESI $\dagger$ ).

To gain insight into the mechanism driving the self-sustained oscillations, a finite element model was developed. We model the LCN as a three-layered system, with individual layers as different alignment domains: homeotropic, intermediate ( $45^{\circ}$ tilt) and planar. The simulations incorporate the temperature response of the network as well as a temperature distribution along the length of the films. In the modelled system, we apply a piecewise linear distribution of the temperature variation as a function of the position of the contact line between the film and the heated surface. Simulations show triangular films to undergo chaotic oscillatory motion with similar tip displacement amplitudes to experimental observations, Fig. 3A and Movie S4 (ESI $\dagger$ ). Furthermore, simulations demonstrate that the location of the contact line rapidly changes during rocking motion, with variation between situation depicted in Fig. 3B (i and ii) being the timescale of one oscillation.

We observe the effective thermal gradient over the film's length varies with shifting location of the contact line at different stages of the rocking motion, Fig. 3B. Zones near the contact line experience large variations in temperature, causing localized thermal strain. Localized cooling in regions becoming distal to the now-shifted contact line leads to strain relaxation in these locations. The variation in temperature and relaxation of thermal strain, primarily in the immediate vicinity of the contact line (note the minimal changes of the tip temperatures during the rocking), leads to different degrees of bending as a function of local temperature (Fig. S4A, ESI $\dagger$ ), unbalancing the bent triangular film and shifting the film's center of gravity. The shifting center of gravity, fueled by local deformation and the location of the contact line results in rocking between two unstable states, iv and vi in Fig. 1C.

Experimentally, we observe that even asymmetric shapes occasionally undergo toppling motion and termination of the feedback-loop. The toppling motion follows an oscillation with unusually high amplitude. It is likely that a threshold value for
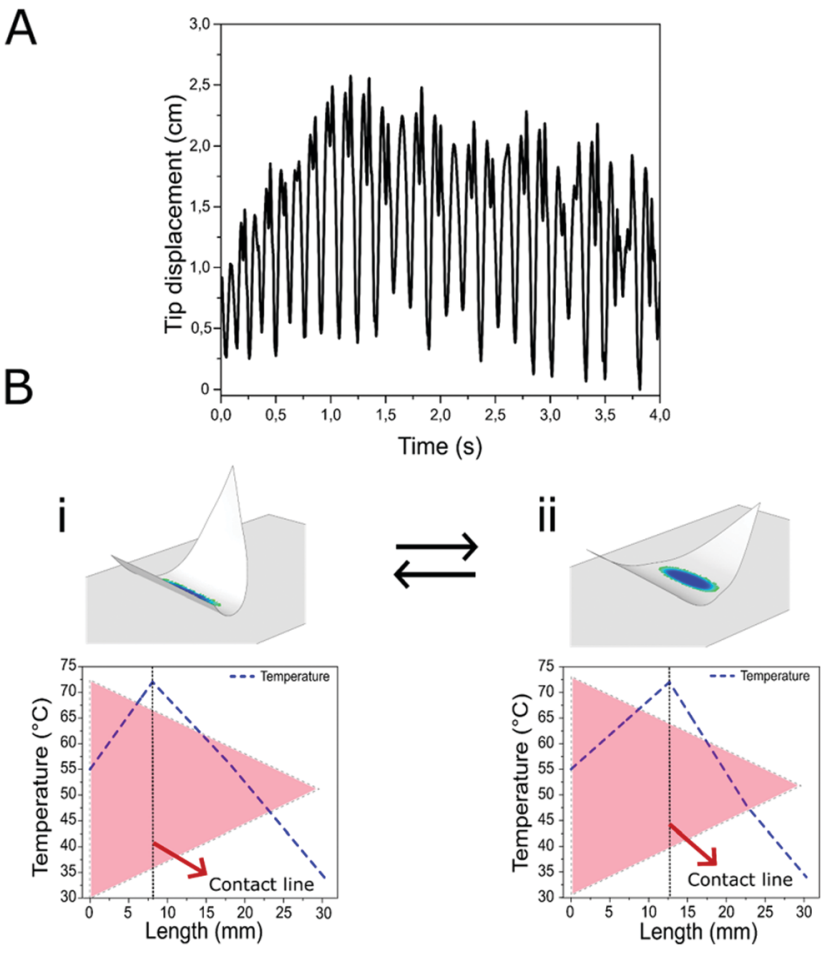

Fig. 3 (A) Plot from tracking the triangular film's tip displacement obtained from the finite element simulation during multiple oscillation cycles. (B) The two depicted positions of the film, i and ii, represent the two displacement extremes during rocking, as shown in Fig. 2B. The plots demonstrate the corresponding temperature profile through the length of the film (blue dashed line) based on the position of the contact line.

oscillation amplitudes $\left(A_{\mathrm{t}}\right)$ exists, which when surpassed causes the film to undergo toppling. The magnitude of the threshold amplitude depends on shape asymmetry; a higher degree of asymmetry shows higher $A_{\mathrm{t}}$ values, allowing for higher amplitude of rocking without toppling. We have simulated a triangle that experiences an oscillation amplitude exceeding $A_{\mathrm{t}}$, and it exhibits a toppling motion similar to objects with lower degrees of asymmetry, such as rectangles or squares, Movie S5 (ESI $\dagger$ ). We propose that for self-sustained oscillations to be maintained, the system must display a unique interplay between shape asymmetry and temperature profile, primarily intimate to the contact region.

A step-wise schematic of mechanical deformations leading to the feedback loop for the sustained oscillations is depicted in Fig. 1C. When the film is placed with the homeotropic surface in contact with the hot plate above $T_{\mathrm{f}}$, it curls, with opposite concavity to its pre-bent shape at room temperature step i and ii in Fig. 1C. This curling causes the extreme edges of the film to lift from the heated surface, and a localized contact region between the film and hot plate is established, creating a thermal gradient along the raised section length Fig. 1C (ii). Upon cooling, these regions experience strain relaxation and change their degree of bending, unbalancing the structure causing tipping towards one side, Fig. 1C (iii). This changes the contact line between the film and hot plate, altering the thermal gradient, causing immediate local heating in this new contact area. Consequently, the area previously heated now cools, Fig. 1C (v), and undergoes an identical sequence 
A

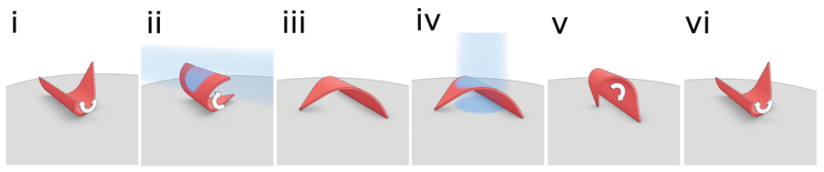

B

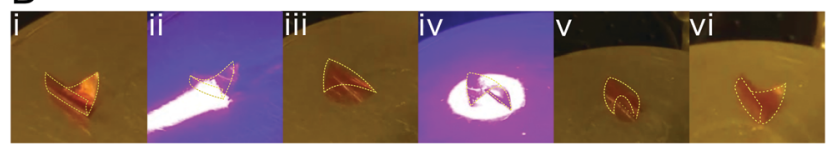

Fig. 4 (A) Schematic of the mechanism for the rock and rolling motion of the triangular LCN triggered by light and the heated surface. (B) Snapshots of the rock and rolling Movie S6 (ESI +$)$; the letters on the snapshots correspond to the schematic description in (A).

of bending events, setting in motion the self-sustained feedback loop, Fig. 1C (iii and iv).

Actuation of the triangular film by light while still on the heated surface results in a film that can be made to rock and additionally roll, with temperature triggering the rocking motion and light activating rolling. Exposure of the LCN to blue light causes localized heating of the film where light is incident, due to the light absorption of the azobenzene dye. The light triggered bending actuation of LCNs doped with such photothermal azobenzenes as dye 3 has been elucidated in previous work. ${ }^{32}$ To demonstrate this additional rolling action, during the rocking motion, a triangular LCN is exposed to a collimated ( $455 \mathrm{~nm}$, corresponding to the absorption of the azobenzene, Fig. S5, ESI $\dagger$ ) light emitting diode (LED) positioned at an oblique angle. This illumination induces the film to begin a directed rolling motion, Fig. 4 and Movie S6 (see ESI $\dagger$ for a full description of the rolling motion). The directed rolling motion results in the displacement of the film over the heated surface.

We disclose a novel, continuous rocking motion of an LCN initiated and sustained simply by a constant heat source located below the device. Via a combination of local actuation, geometric shape, and thermal gradients, various bending directions are found within proximity in the same film, resulting in continual overbalancing of the structure, and manifested as a self-sustained rocking motion. Through finite element simulations, we have shown that the sustained oscillations are geometry dependent and that temperature specific deformations propel the oscillatory motion. By further including a photo-responsive molecule, the actuator can be made to rock and roll, showing that the combination of dual responsiveness in a simple device can result in the generation of complex motions which can be manipulated and self-sustained.

\section{Conflicts of interest}

There are no conflicts to declare.

\section{Notes and references}

1 X. Lu, S. Guo, X. Tong, H. Xia and Y. Zhao, Adv. Mater., 2017, 29, 1-7.

2 S. I. Rich, R. J. Wood and C. Majidi, Nat. Electron, 2018, 1, 102-112.

3 R. Merindol and A. Walther, Chem. Soc. Rev., 2017, 46, 5588-5619.

4 H. Arazoe, D. Miyajima, K. Akaike, F. Araoka, E. Sato, T. Hikima, M. Kawamoto and T. Aida, Nat. Mater., 2016, 15, 1084-1089.

5 Y. Liu, B. Xu, S. Sun, J. Wei, L. Wu and Y. Yu, Adv. Mater., 2017, 29, 1604792.

6 S. Serak, N. Tabiryan, R. Vergara, T. J. White, R. A. Vaia and T. J. Bunning, Soft Matter, 2010, 6, 779-783.

7 X. He, M. Aizenberg, O. Kuksenok, L. D. Zarzar, A. Shastri, A. C. Balazs and J. Aizenberg, Nature, 2012, 487, 214-218.

8 M. L. Smith, C. Slone, K. Heitfeld and R. A. Vaia, Adv. Funct. Mater., 2013, 23, 2835-2842.

9 H. Zhou, Z. Zheng, Q. Wang, G. Xu, J. Li and X. Ding, RSC Adv., 2015, 5, 13555-13569.

10 M. Yamada, M. Kondo, J. I. Mamiya, Y. Yu, M. Kinoshita, C. J. Barrett and T. Ikeda, Angew. Chem., Int. Ed., 2008, 47, 4986-4988.

11 T. Ikeda, J. I. Mamiya and Y. Yu, Angew. Chem., Int. Ed., 2007, 46, 506-528.

12 G. N. Mol, K. D. Harris, C. W. M. Bastiaansen and D. J. Broer, Adv. Funct. Mater., 2005, 15, 1155-1159.

13 O. M. Wani, H. Zeng and A. Priimagi, Nat. Commun., 2017, 8, 15546. 14 J. A. H. P. Sol, A. R. Peeketi, N. Vyas, A. P. H. J. Schenning, R. K. Annabattula and M. G. Debije, Chem. Commun., 2019, 55, 1726-1729.

15 J. J. Wie, M. R. Shankar and T. J. White, Nat. Commun., 2016, 7,13260 .

16 A. H. Gelebart, D. Jan Mulder, M. Varga, A. Konya, G. Vantomme, E. W. Meijer, R. L. B. Selinger and D. J. Broer, Nature, 2017, 546, 632-636.

17 M. Rogóż, H. Zeng, C. Xuan, D. S. Wiersma and P. Wasylczyk, Adv. Opt. Mater., 2016, 4, 1689-1694.

18 M. Yamada, M. Kondo, R. Miyasato, Y. Naka, J. I. Mamiya, M. Kinoshita, A. Shishido, Y. Yu, C. J. Barrett and T. Ikeda, J. Mater. Chem., 2009, 19, 60-62.

19 S. J. Aßhoff, F. Lancia, S. Iamsaard, B. Matt, T. Kudernac, S. P. Fletcher and N. Katsonis, Angew. Chem., Int. Ed., 2017, 56, 3261-3265.

20 R. C. P. Verpaalen, M. G. Debije, C. W. M. Bastiaansen, H. Halilović, T. A. P. Engels and A. P. H. J. Schenning, J. Mater. Chem. A, 2018, 6, 17724-17729.

21 C. L. Van Oosten, K. D. Harris, C. W. M. Bastiaansen and D. J. Broer, Eur. Phys. J. E: Soft Matter Biol. Phys., 2007, 23, 329-336.

22 H. K. Bisoyi and Q. Li, Chem. Rev., 2016, 116, 15089-15166.

23 M. Yang, Z. Yuan, J. Liu, Z. Fang, L. Fang, D. Yu and Q. Li, Adv. Opt. Mater., 2019, 7, 1900069.

24 L. T. De Haan, J. M. N. Verjans, D. J. Broer, C. W. M. Bastiaansen and A. P. H. J. Schenning, J. Am. Chem. Soc., 2014, 136, 10585-10588.

25 Q. Chen, X. Yan, H. Lu, N. Zhang and M. Ma, ACS Appl. Mater. Interfaces, 2019, 11, 20473.

26 T. J. White, N. V. Tabiryan, S. V. Serak, U. A. Hrozhyk, V. P. Tondiglia, H. Koerner, R. A. Vaia and T. J. Bunning, Soft Matter, 2008, 4, 1796.

27 A. H. Gelebart, G. Vantomme, E. W. Meijer and D. J. Broer, Adv. Mater., 2017, 29, 1606712.

28 F. Ge, R. Yang, X. Tong, F. Camerel and Y. Zhao, Angew. Chem., Int. Ed., 2018, 57, 11758-11763.

29 K. Kumar, C. Knie, D. Bléger, M. A. Peletier, H. Friedrich, S. Hecht, D. J. Broer, M. G. Debije and A. P. H. J. Schenning, Nat. Commun., 2016, 7, 11975.

30 F. Ge and Y. Zhao, Chem. Sci., 2017, 8, 6307-6312.

31 C. R. Bowen, J. Taylor, E. Leboulbar, D. Zabek, A. Chauhan and R. Vaish, Energy Environ. Sci., 2014, 7, 3836-3856.

32 M. Pilz da Cunha, Y. Foelen, T. A. P. Engels, K. Papamichou, M. Hagenbeek, M. G. Debije and A. P. H. J. Schenning, Adv. Opt. Mater., 2019, 7, 1801604. 